\title{
Structure and Hydrogen Bonds of Hydrophobic Deep Eutectic Solvent-Aqueous Liquid-Liquid Interfaces
}

\author{
Usman Abbas ${ }^{1}$, Qi Qiao ${ }^{1}$, Manh Tien Nguyen ${ }^{1}, \mathrm{Jian}_{\mathrm{Shi}^{2}}$, and Qing Shao ${ }^{1}$ \\ ${ }^{1}$ University of Kentucky \\ ${ }^{2}$ Affiliation not available
}

May 26, 2021

\begin{abstract}
Hydrophobic deep eutectic solvents (DESs) emerge as candidates to extract organic substrates from aqueous solutions. The DESaqueous liquid-liquid interface plays a vital role in the extraction ability of hydrophobic DES because the non-bulk structure of molecules at the interface could cause thermodynamic and kinetic barriers. One question is how the DES compositions affect the structural features of the DES-aqueous liquid-liquid interface. We investigate the density profile, dipole moment and hydrogen bonds of eight hydrophobic DES-aqueous liquid-liquid interfaces using molecular dynamics simulations. The eight DESs are composed of four organic compounds: decanoic acid, menthol, thymol, and lidocaine. The simulation results show the variations of dipole moment and hydrogen bond structure and dynamics at the liquid-liquid interfaces. Such variations could influence the extraction ability of DES through adjusting the partition and kinetics of organic substrates in the DES-aqueous biphasic systems.
\end{abstract}

\section{Hosted file}

Interfacial properties of hydrophobic DESs-QQ-5-12.docx available at https://authorea.com/ users/396416/articles/523712-structure-and-hydrogen-bonds-of-hydrophobic-deep-eutecticsolvent-aqueous-liquid-liquid-interfaces 